\title{
"HN Turn to Drive Osteotome": A Customized Device for Preparation of Osteotomy for Implant Placement
}

\author{
${ }^{1}$ Karunakaran Harshakumar, ${ }^{2}$ Nimisha B Abraham
}

\begin{abstract}
The density of available bone in an edentulous site has a primary influence on the treatment planning and the surgical approach chosen for osteotomy. In situations of inadequate bone density, osteotomes are a special set of hand instruments developed to form and shape bone, in preparation to placement of dental implants. Conventional osteotomes are optimally used by pressing the instruments into the bone and malleting into place using a surgical mallet. This article describes a variant of the conventional osteotome, which was indigenously developed to use the handdriven force for implant placement and, henceforth, eliminate the drawbacks of malleting procedures. This would aid hassle-free implant placement in edentulous areas with compromised bone quality and quantity.
\end{abstract}

Keywords: Hand driven, Implant bed preparation, Osteotome.

How to cite this article: Karunakaran H, Abraham NB. "HN Turn to Drive Osteotome": A Customized Device for Preparation of Osteotomy for Implant Placement. Int J Oral Implantol Clin Res 2017;8(1):1-4.

\section{Source of support: Nil}

Conflict of interest: None

\section{INTRODUCTION}

Bone healing subsequent to osteotomy for housing an implant is critical for implant success. The surgical process for bone osteotomy consists of drilling a pilot aperture and its subsequent widening either by conventional bone drilling or osteotome techniques.

Conventional bone-drilling technique uses drills of various diameters in a sequential manner to remove bone from the implant site and widen the site diameter until the final drill meets the implant diameter. However, implant placement with drills in relatively less dense bone leads to heat generation, loss of bone, enlargement or elliptical preparation of the osteotomy site, loss of tactile sensitivity, sinus penetration, and overpreparation of soft bone.

\footnotetext{
${ }^{1}$ Professor and Head, ${ }^{2}$ Postgraduate Student

1,2Department of Prosthodontics, Government Dental College Thiruvananthapuram, Kerala, India
}

Corresponding Author: Nimisha B Abraham, Postgraduate Student, Department of Prosthodontics, Government Dental College, Thiruvananthapuram, Kerala, India, Phone: +917293683574, e-mail: newnimi@gmail.com
A technique with conventional osteotomes was put forward by various proponents to tackle the demerits attributed to the osteotomy by conventional drilling. ${ }^{1}$ Osteotomes are a special set of hand instruments that sculpt the bone in preparation for placement of dental implants. Conventional osteotomes are used optimally by pressing the instruments into the bone and malleting into place using a surgical mallet. This technique of preparation of the implant site involves drilling of a pilot aperture followed by use of multiple osteotomes (with a mallet and chisel technique) to enlarge the implant site without further removal of bone. This attempts to retain all the bone present and take advantage of the softer bone quality by relocating the bone to form a denser bone interface with the implant. Osteotome technique allows for minimal heat generation, greater tactile sensitivity, thereby preventing elliptical osteotomy preparation and expansion of the narrow ridge. It is preferable to prepare the implant site through the use of osteotomes as it preserves bone, rather than by bone removal according to the drilling technique.

Despite these advantages, conventional osteotomes still possess some demerits, as they cause traumatic expansion due to percussion and produce paroxysmal vertigo due to labyrinthine concussion that usually lasts for 2 or 3 days because of the impact produced by the surgical mallet. ${ }^{1}$ Additionally, hammered osteotomes often become embedded in the bone and require considerable force to remove. Despite the proper alignment of the pilot hole, alignments of the implantation site are prone to shift as the osteotomes are hammered manually. Moreover, osteotomes demand need of two operators, one to support the osteotome and the other to do the percussion, which may cause intrusion in compromised areas, such as the infraorbital or into the maxillary sinus.

The "HN turn to drive osteotome" is indigenously developed and fabricated in the Department of Prosthodontics, Government Dental College, Thiruvananthapuram, India, to overcome these demerits. These osteotomes are hand driven eliminating the drawbacks of malleting procedures. The instrument was then used to prepare the implant bed in the less dense bone without the need for a surgical mallet.

\section{MATERIALS AND METHODS}

The instrument was fabricated by milling a medical grade 304 stainless steel. Stainless steel 304 offers a number of 
advantages, such as high corrosion resistance, recyclable and antibacterial properties; it is also nonstainable.

\section{Parts}

The osteotome is designed with five parts (Fig. 1).

1. Handle

This consists of a vertical arm perpendicular to horizontal cross-arm making a $\mathrm{T}$. The vertical arm connects the horizontal arm to the rest of the instrument. The vertical arm is designed with $2.5-\mathrm{mm}$-wide bands located at a constant interval making the surface of the vertical arm irregular. These bands along with the cross-arm aid in the positioning of the osteotome and provide good grip while instrumenting. An extension of the vertical arm projects out at the $\mathrm{T}$ junction, which is a provision for malleting as in the conventional osteotome.

2. Shank

It is bi-angled and connects the handle to the other parts of the osteotome. This design enables easy access to the maxillary posterior region and prevents any interference with the lip.

3. Bottom Lock Screw

This functions as a stop for correctly positioning the length adjustment screw at the desired length, as it

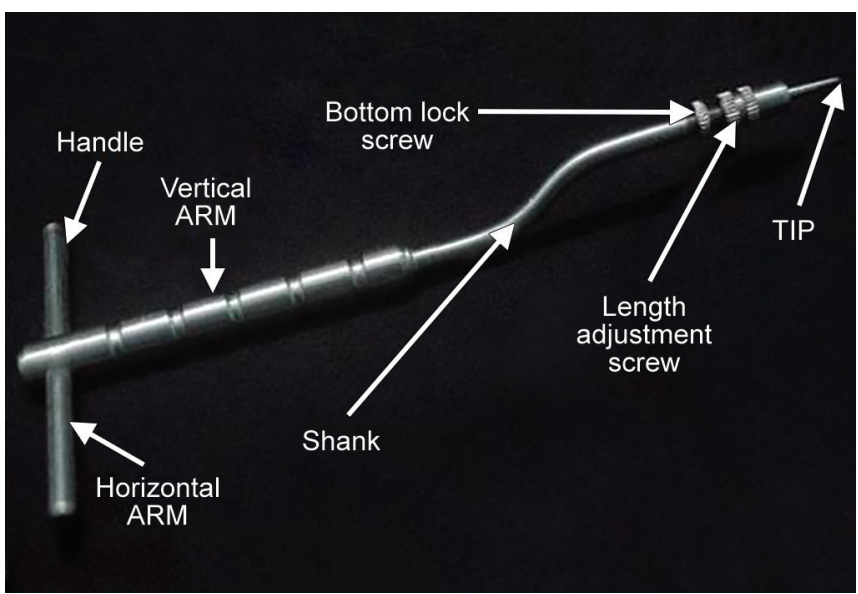

Fig. 1: Parts of an osteotome

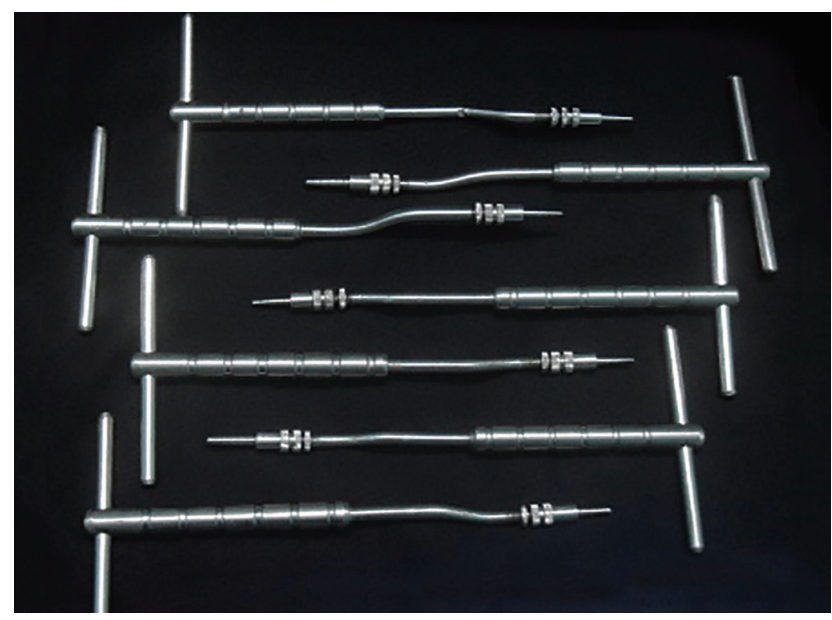

Fig. 3: Osteotomes of subsequently larger diameters can be moved superiorly and inferiorly and locked at desired length.

4. Length Adjustment Screw

This screw can be moved superiorly and inferiorly along the length of the tip of the instrument and locked to the marking corresponding to the length of the implant.

5. Tip

The tip is narrow and tapering with calibrations at 2, 5, $8,11,13$, and $16 \mathrm{~mm}$ (Fig. 2). Eight subsequently larger tip diameters are available, i.e., 1, 2.0, 2.5, 2.8, 3.0, 3.2, 4.3, and $5.2 \mathrm{~mm}$ (Fig. 3).

\section{Procedure}

- Supracrestal incision is made and soft tissue flap is raised. Drill pilot aperture at the edentulous area up to the predetermined depth corresponding to the implant length (Figs 4 to 6).

- Position the osteotome corresponding to the longitudinal axis of the missing teeth or the adjacent teeth each time it is manipulated.

- Set the desired length in the osteotome with length adjustment screw and bottom lock screw.

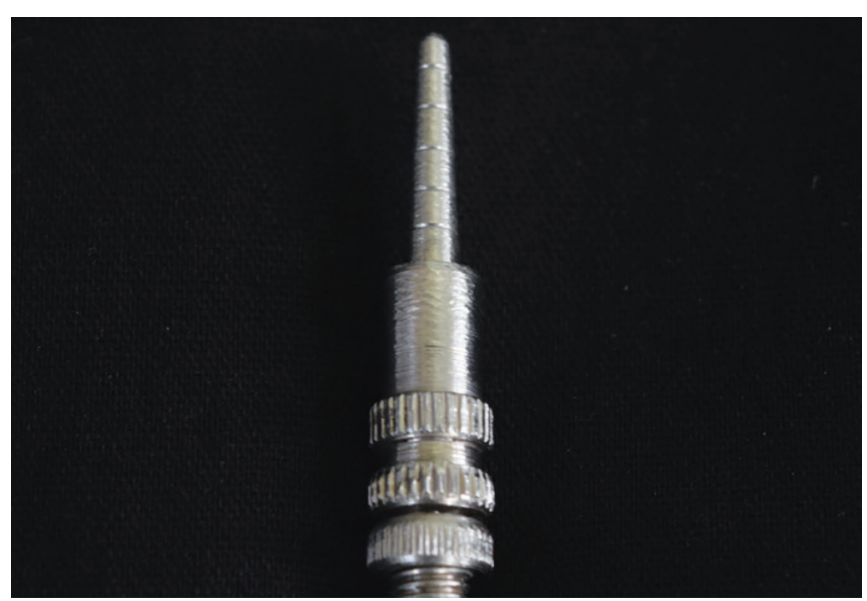

Fig. 2: Tip of the osteotomes showing callibrations

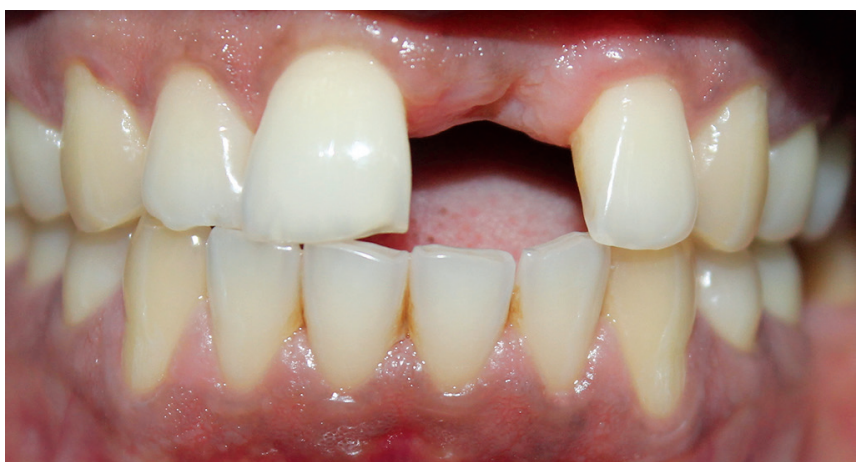

Fig. 4: Preoperative intraoral frontal view showing missing central incisor 


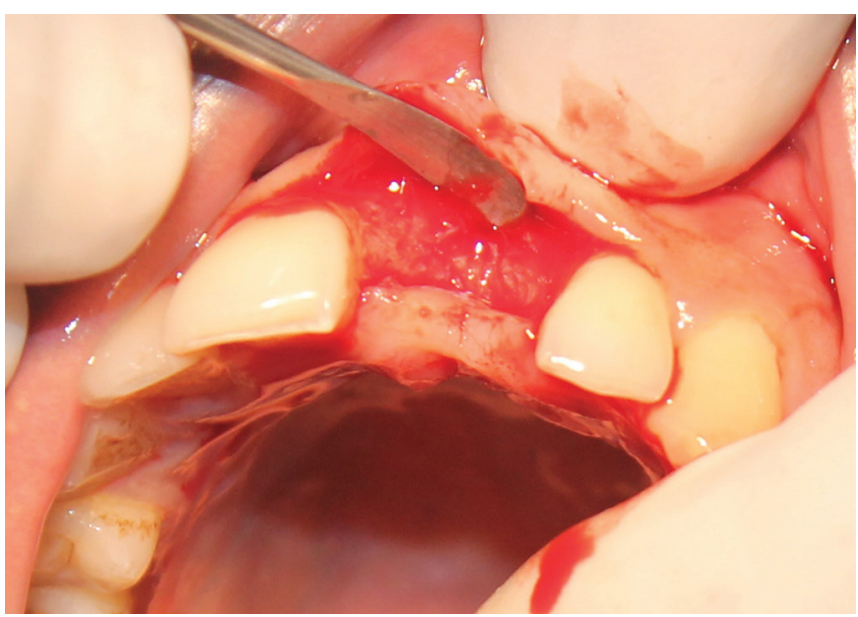

Fig. 5: Flap reflection

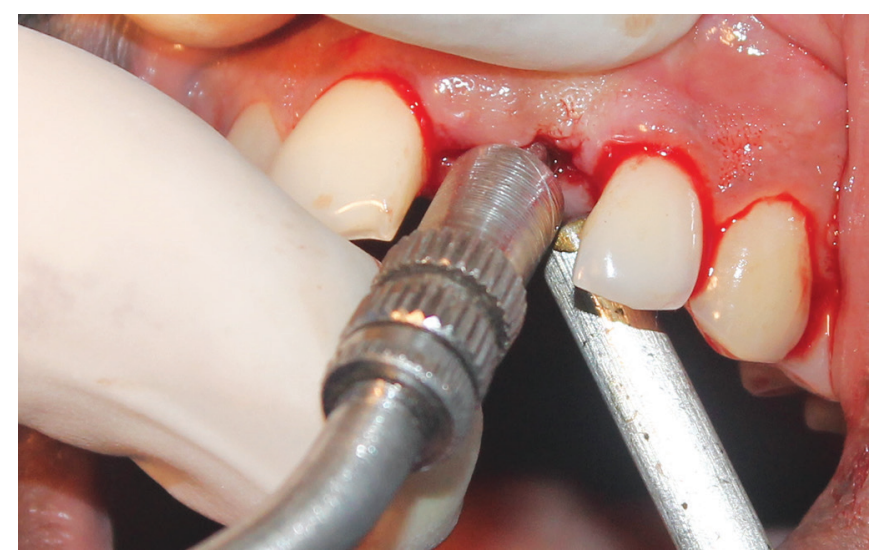

Fig. 7: Use of "HN turn to drive osteotome" for further widening of osteotomy

- Enlarge the osteotome site by turning the 2-mm osteotome in a clockwise direction (Fig. 7).

- Do a quarter circle turn of the osteotome each time. This sequence is continued until the predetermined depth is attained.

- Size the osteotome site with successively larger osteotomes until the desired diameter that corresponds to the implant is reached.

- Insert the implant into the implant bed (Fig. 8).

\section{DISCUSSION}

Osteotomy is invariably performed using the conventional bone-drilling technique by most clinicians. It was found that implant survival in initial surgical success was related to the quality of bone by following the proposed protocols of Adell et al. ${ }^{2}$ Placement of implants with drills presented with a set of surgical problems in relatively less dense bone. ${ }^{3}$ These include heat generation, loss of bone, enlargement or elliptical preparation of the osteotomy site, loss of tactile sensitivity, sinus penetration, and overpreparation of soft bone. On the contrary, osteotomes are surgical instruments that can be used effectively to enhance the quality of less dense bone, especially in the maxillary arch. The

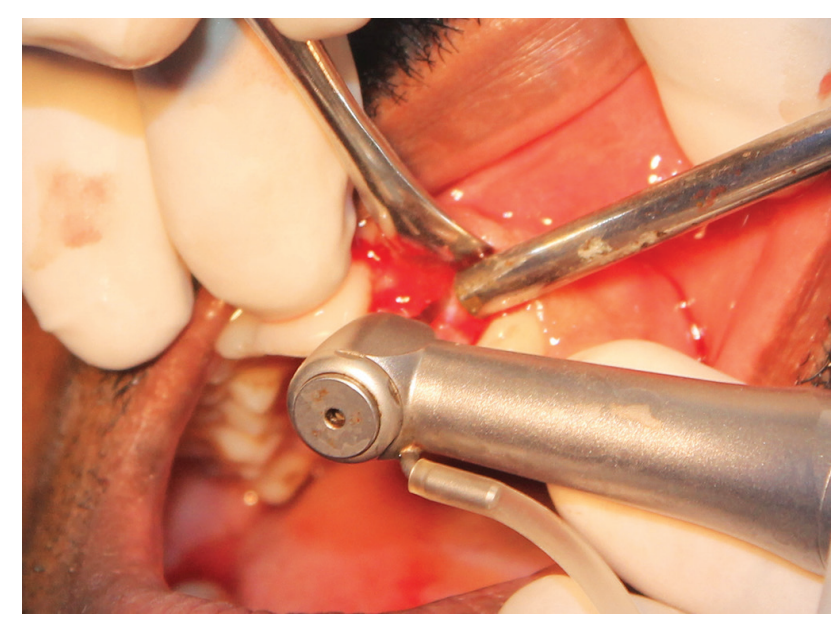

Fig. 6: Initial osteotomy using pilot drill

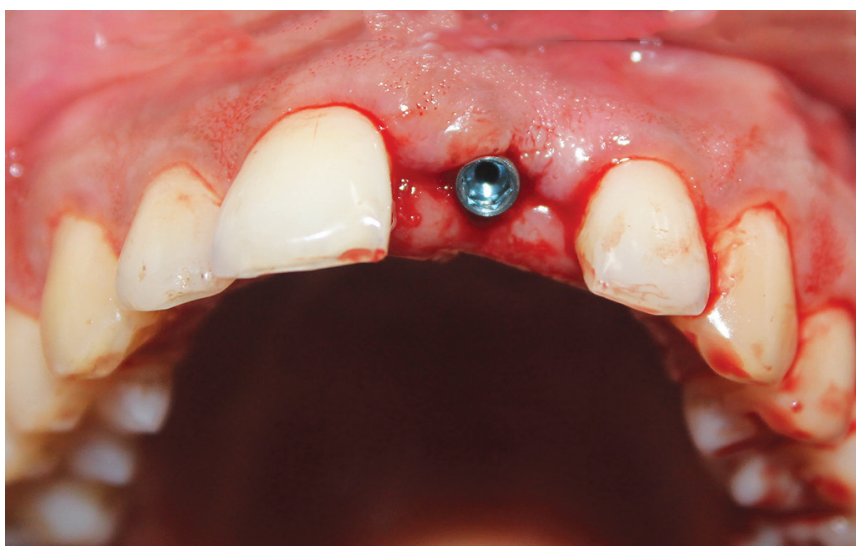

Fig. 8: Implant in situ

advocates of osteotome technique put forth the advantages like compression and relocation of bone, thereby converting softer bone to a denser one; minimal heat generation; improved tactile sensitivity; and enhanced primary stability by using the osteotomes for implant bed preparation. ${ }^{3-8}$ All these above-mentioned advantages make the osteotome technique increasingly popular, especially for implants in the maxillary arch. Patient comfort is a major factor not met while using osteotomes due to surgical malleting. The consequences of surgical malleting include headache, paroxysmal vertigo, etc. Also, the need for two operators is inevitable. There arises the need for this custom-modified "HN turn to drive osteotome," which precludes all the above-mentioned adverse effects. The design considerations help the operator to handle the instrument with ease, especially less dense bone types with minimal discomfort to the patient. Additional provision for malleting in the handle can aid in indirect sinus lift situations.

\section{CONCLUSION}

Osteotomes are important adjunctive instruments during dental implant placement. The "HN turn to drive osteotomes" are more efficient and highly oriented with regard to patient comfort than the conventional osteotomes. They 
are economical, less traumatic, and less time-consuming. They have a simple design and yet deliver the working force effectively. The technique is gentle, as it requires only human-generated force and does not generate heat; it also gives the surgeon a tactile sense of bone quality.

\section{REFERENCES}

1. Summers RB. A new concept in maxillary implant surgery: the osteotome technique. Compendium (Newtown, Pa.) 1994 Feb;15(2):152-154.

2. Adell R, Lekholm U, Rockler BR, Brånemark PI. A15-year study of osseointegrated implants in the treatment of the edentulous jaw. Int J Oral Surg 1981 Jan;10(6):387-416.

3. Siddiqui AA, Sosovicka M. Lateral bone condensing and expansion for placement of endosseous dental implants: a new technique. J Oral Implantol 2006 Apr;32(2):87-94.
4. De Vico G, Bonino M, Spinelli D, Pozzi A, Barlattani A. Clinical indications, advantages and limits of the expansion-condensing osteotomes technique for the creation of implant bed. Oral Implantol 2009 Jan;2(1):27.

5. Hahn J. Clinical uses of osteotomes. J Oral Implantol 1999 Jan;25(1):23-29.

6. Krafft T, Graef F, Winter W, Wichmann M, Karl M. Use of osteotomes for implant bed preparation-effect on material properties of bone and primary implant stability. J Oral Implantol 2013 May;39(s1):241-247.

7. Marković A, Ćalasan D, Čolić S, Stojčev-Stajčić L, Janjić B, Mišić T. Implant stability in posterior maxilla: bone-condensing versus bone-drilling: a clinical study. Oral Surg Oral Med Oral Pathol Oral Radiol Endodontol 2011 Nov;112(5):557-563.

8. Ferrer JR, Diago MP, Carbó JG. Analysis of the use of expansion osteotomes for the creation of implant beds. Technical contributions and review of the literature. Med Oral Patol Oral Cir Bucal 2006 May;11(3):E267-E271. 